\title{
PROCEDIMENTO PARA AVALIAÇÃO DO IMPACTO DE NOVAS LINHAS DE ÔNIBUS EM TERMOS DE ACESSIBILIDADE A HOSPITAIS AUXILIADO POR SISTEMA GIS
}

\author{
Gustavo Patrocinio Molino \\ Universidade Federal de Viçosa, Departamento de Engenharia de Produção e Mecânica \\ $\mathrm{Ph}$. Rolfs s/n - 36570-000 - Viçosa - MG \\ gustavo.pmolino@gmail.com
}

\section{INTRODUÇÃO}

Mesmo com o passar dos anos e com o desenvolvimento tecnológico, as áreas urbanas ainda sofrem com os problemas vistos no início dos anos 2000, como o acentuado crescimento populacional e, por consequência, os congestionamentos de automóveis (Schrank, 2015). Um dos diversos impactos sentidos pela população de uma cidade e que é causado pelos congestionamentos urbanos é o acesso a serviços de saúde. Nesse cenário, o transporte é considerado uma das principais barreiras ao um rápido atendimento médico (Syed et al. 2005).

Como outros grandes centros, a cidade de Chicago, E.U.A, enfrenta problemas quanto ao seu sistema público de transporte. Neste sentido, buscando aumentar a eficiência de seu sistema, diversos projetos surgem, como por exemplo, a extensão da rota 11, a criação do "Loop link" e a criação linha Ashland BRT (Chicago transit authority, 2016). O Ashland BRT, consiste em uma linha de ônibus que percorreria a Avenida Ashland, cruzando a cidade de norte a sul e conectaria importantes localidades como o distrito médico de Illinois, a universidade de Illinois em Chicago, a escola Malcom X e o United Center.

No entanto, outra questão surge, qual será o impacto desses projetos, em termos de acesso a serviços de saúde. Dessa forma o presente estudo busca estimar o impacto da criação da linha Ashland BRT na malha de transporte público da cidade de Chicago, E.U.A, em termos de acessibilidade a hospitais.

\section{METODOLOGIA}

A metodologia utilizada nesse trabalho consiste em oito etapas, descritas a seguir:

1. Levantamento de dados;

2. Construção da malha virtual referente à atual malha de transporte público utilizando software GIS;

3. Obtenção da matriz origem-destino do cenário atual;

4. Estimativa do tempo de deslocamento necessário atualmente;

5. Construção da malha virtual referente à malha de transporte público proposta utilizando software GIS;

6. Obtenção da matriz origem-destino do cenário proposto;

7. Estimativa do tempo de deslocamento necessário no cenário proposto;

8. Comparação dos cenários.

Para a realização do estudo foi necessário obter o mapa dos pontos de concentração populacional (800 pontos), o mapa dos logradouros, o mapa dos hospitais (37 hospitais) e a 
capacidade de atendimento dos mesmos, o feed GTFS (especificação geral de feeds de transporte público) da cidade de Chicago e o projeto detalhado da linha em estudo.
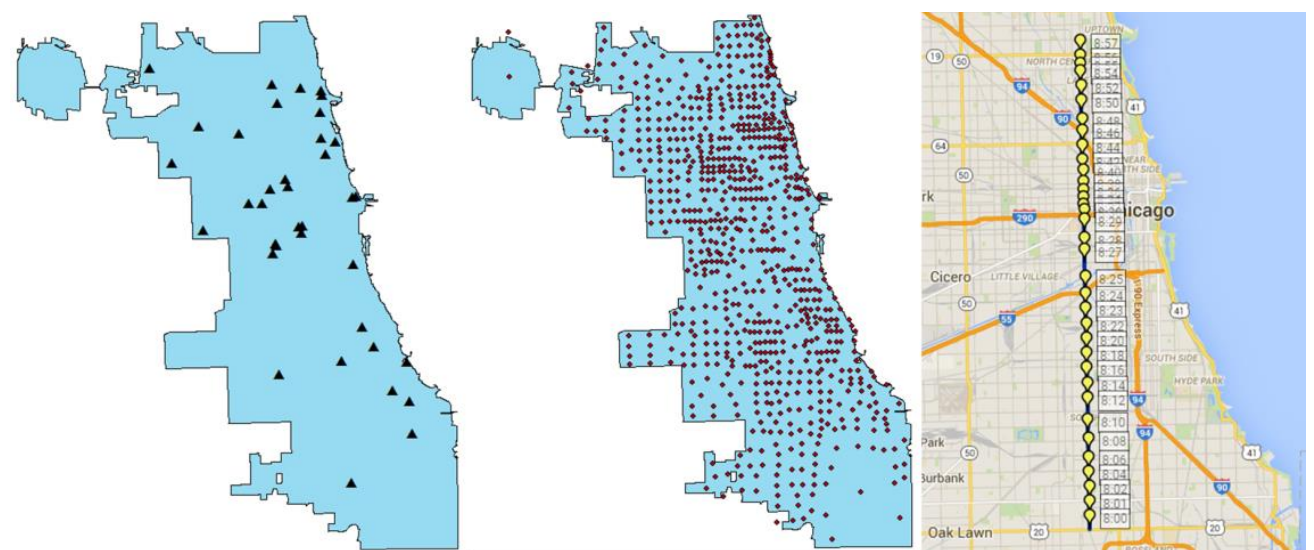

Figura 1 - Localização dos hospitais (à esquerda; triângulos), pontos de concentração populacional (centro; quadrados) e linha Ashland BRT (à direita; linha azul conectada por círculos amarelos).

Após a obtenção dos dados, os mesmos foram inseridos no software ArcGIS, utilizando as caixas de ferramentas network analyst toolbox e "Add GTFS to a Network Dataset", disponível no website "Yay, transit!", visando obter a matriz origem-destino.

A terceira etapa tem como objetivo atribuir grupos populacionais aos hospitais. Para tal, o modelo de matemático descrito abaixo foi elaborado combinando o clássico problema do transporte e o modelo de Huff (Huff,1964). Após a elaboração, o modelo foi implementado no software AMPL. Os tempos de deslocamento (t) são fornecidos pela matriz origem-destino.

$$
\begin{aligned}
& \text { Maximizar } \sum_{i=1}^{P C P} \sum_{j=1}^{H}\left(\left(\left(c_{j} / t_{i, j}\right) \times\left(X_{i, j}\right)\right) /\left(\sum_{k=1}^{H} c_{k} / t_{i, k}\right)\right) \\
& \sum_{j=1}^{H} X_{i, j}=p_{i}, \forall i \\
& \sum_{i=1}^{P C P} X_{i, j} \leq c_{j}, \forall j
\end{aligned}
$$

Sendo as variáveis de decisão e os parâmetros utilizados no modelo os seguintes:

- $\quad P C P$ : o número de pontos de concentração populacional;

- $\quad p_{i}$ : população do ponto de concentração populacional $i$;

- $H$ : o número de hospitais;

- $\quad c_{j}$ : a capacidade do hospital $j$;

- $\quad c_{k}$ : a capacidade do hospital $k$;

- $t_{i, j}$ : tempo de deslocamento entre o ponto de concentração populacional $i$ e o hospital $j$;

- $t_{i, k}$ : tempo de deslocamento entre o ponto de concentração populacional $i$ e o hospital $k$;

- $X_{i, j}$ : variável de decisão que representa o número de pessoas do ponto de concentração populacional $i$ que é atendida pelo o hospital $j$;

Para a compreensão da função objetivo, Eq. (1), o conceito de atratividade deve ser definido. A atratividade de ponto de concentração populacional $i$ por um hospital $j$ é definida como a razão entre a capacidade do hospital $j$ e o tempo de deslocamento entre o ponto de concentração populacional $i$ e o hospital $j$. Em outras palavras, a atratividade é diretamente proporcional à capacidade do hospital e inversamente proporcional ao tempo de deslocamento.

Dessa forma, através da função descrita, o modelo visa alocar os pontos de concentração populacional aos hospitais de maior atratividade. 
Nesse modelo duas restrições são exigidas. A primeira, Eq. (2), garante que toda a população de uma concentração populacional seja atendida por um ou mais hospitais. A segunda, Eq. (3) garante que nenhum hospital ultrapasse sua capacidade.

Ao fim desta etapa, a partir do resultado do modelo e dos dados da matriz origem-destino, o tempo de deslocamento que cada pessoa necessita atualmente para chegar ao hospital é obtido.

$\mathrm{Na}$ etapa cinco, uma nova malha virtual é construída. Entretanto, neste caso a linha proposta foi incorporado no feed GTFS.

Em sequência, as etapas seis e sete são realizadas de forma análoga as etapas três e quarto, respectivamente.

Com os resultados obtidos nas etapas quatro e sete, os cenários foram comparados através de uma análise quantitativa.

\section{RESULTADOS}

Com a adição da linha Ashland BRT, 1.104 .510 pessoas foram afetadas, $41 \%$ da população considerada. Desse total, $79 \%$ tiveram seu tempo de deslocamento reduzido, sendo 38,8673 minutos a maior redução identificada e 33,4082 minutos o maior acréscimo. No geral, o tempo médio de acesso ao hospital que cada grupo populacional foi alocado reduziu 1,428 minutos, de 31,605 minutos para 30,177 minutos.

\section{DISCURSÃO}

Podemos verificar que a adição de novas linhas também geraram impactos negativo, em termos de acessibilidade a hospitais. Esse impacto negativo é consequência da capacidade física dos hospitais. Com a adição da nova linha, os paramentos de atratividade sofrem alterações, forçando certos grupos populacionais para hospital mais distante, visto que os mais próximos já estarão em lotação máxima.

\section{CONCLUSÃO}

Podemos concluir que o projeto Ashland BRT, possui um impacto positivo para a cidade de Chicago, E.U.A. No entanto, esse estudo isoladamente não é suficiente para dizer que esse projeto em questão é melhor, em termos de impacto, que outros potenciais projetos. Com o intuito de realizar uma classificação dos projetos é recomentado que todos sejam submetidos a esse procedimento.

\section{AGRADECIMENTOS}

Agradeço ao professor Hemanshu Kaul, pela orientação nesse trabalho. Ao estudante Ulisses Américo Carvalho Oliveira, pela sua fundamental contribuição nesse trabalho, e também aos estudantes Iago Carrara Roque e João Paulo Gusmão Teixeira pela assistência prestada na redação desse resumo.

\section{REFERÊNCIAS}

CHICAGO TRANSIT AUTHORITY, 2016. “Improvement Projects”. Acesso em: 24 out. 2016. Disponível em: < http://www.transitchicago.com/news_initiatives/projects/default.aspx >

SCHRANK, D., EISELE, B., LOMAX, T., BAK, J. "Urban mobility scorecard”. The Texas A\&M Transportation Institute and INRIX. Texas. Ago. 2015

SYED, S. T., GERBER, B. S., \& SHARP, L. K. "Traveling towards disease: transportation barriers to health care access". Journal of community health (2005). v. 38, n. 5, p. 976-993, out. 2013

HUFF, D.L. "Defining and estimating a trading area". The Journal of Marketing. v. 28, n. 3, p. 34 -38 , jul. 1964. 